\title{
Haploidentical hematopoietic stem cell transplantation with one-day posttransplant cyclophosphamide and anti-thymocyte globulin for graft failure
}

\author{
Hiroshi Imanaga', Takanori Ohta², Seido Oku², Yasuhiro Sugio², Yuju Ohno \\ ${ }^{1}$ Medicine and Biosystemic Science, Kyushu University Graduate School of Medical Sciences, Fukuoka, Japan, ${ }^{2}$ Depart- \\ ment of Internal Medicine, Kitakyushu Municipal Medical Center, Fukuoka, Japan
}

\begin{abstract}
Patients with myelofibrosis usually have poor prognosis. Allogeneic hematopoietic stem cell transplantation (allo-HSCT) remains the only curative therapy, which has graft failure as a life-threatening complication. However, no consensus is available with regard to therapeutic options for patients with graft failure. Here we report a patient with myelofibrosis who underwent successful salvage haploidentical hematopoietic stem cell transplantation (haplo-HSCT) with one-day posttransplant cyclophosphamide (PTCy) and low-dose anti-thymocyte globulin (ATG) for graft failure. A 39-year-old Japanese male patient with rapidly progressing primary myelofibrosis underwent cord blood transplantation (CBT). Unfortunately, both the first and second CBT resulted in primary graft failures. Therefore, emergent haplo-HSCT from a sibling donor was performed with one-day PTCy (50 mg/ $\mathrm{kg}$ on day +3$)$ after conditioning with etoposide $\left(60 \mathrm{mg} / \mathrm{m}^{2}\right.$ on days -3 and -2$)$ and rabbit anti-human thymocyte globulin $(1 \mathrm{mg} / \mathrm{kg}$ on days -2 and -1$)$. Neutrophil engraftment was achieved on day +13 after haplo-HSCT, and no severe infection or regimen-related toxicity was observed. Skin stage 3, gut stage 1 total grade $I$ acute graft-versus-host disease developed. His posttransplant course had been uneventful with cutaneous chronic graftversus-host disease (NIH score 2) and suppressed relapse. We believe that haplo-HSCT with one-day PTCy and lowdose ATG is one of the successful therapeutic options for graft failure.
\end{abstract}

Key words: graft failure, haploidentical hematopoietic stem cell transplantation, anti-thymocyte globulin, posttransplant cyclophosphamide, primary myelofibrosis

Submitted July 19, 2019; Accepted December 15, 2019; Published online April 21, 2020; Issued online May 25, 2020

Correspondence: Hiroshi Imanaga, Medicine and Biosystemic Science, Kyushu University Graduate School of Medical Sciences, Fukuoka, Japan 3-1-1 Maidashi, Higashi-ku, Fukuoka, 812-8582, Japan, E-mail: imanaga. hiroshi.967@m.kyushu-u.ac.jp

\section{Introduction}

Myelofibrosis is a pathological condition caused by the clonal proliferation of a pluripotent stem cell and is characterized by deregulated kinase signaling and abnormal cytokine release. Myelofibrosis is associated with three main driver mutations of $J A K 2, C A L R$, and $M P L^{1}$.

Despite recent developments in targeted therapies (JAK 1/2 inhibitors), patients diagnosed with myelofibrosis usually have poor prognosis, and allogeneic hematopoietic stem cell transplantation (allo-HSCT) remains the only curative therapy. However, allo-HSCT is always associated with graft failure, particularly in cord blood transplantation $(\mathrm{CBT})^{2,3}$. Currently, the number of patients undergoing haploidentical hematopoietic stem cell transplantation (haplo-HSCT) with posttransplant cyclophosphamide (PTCy) has been increasing. However, salvage haplo-HSCT with PTCy performed for graft failure remains controversial, and the appropriate conditioning regimen and optimal PTCy dose remain unclear. Herein, we report a patient with primary myelofibrosis (PMF) successfully engrafted by haplo-HSCT with oneday PTCy and low-dose anti-thymocyte globulin (ATG) after graft failure following CBT.

\section{Case Presentation}

A 39-year-old Japanese male patient diagnosed with PMF presented with polyarthralgia and splenomegaly. His peripheral blood examination revealed a white blood 
Table 1. Summary of HSCTs in our case

\begin{tabular}{lccc}
\hline & 1st transplantation & 2nd transplantation & 3rd transplantation \\
\hline Donor type/sex & MUD/male & MisUD $/$ male & MisRD (haplo) $/$ male \\
Stem cell source & CB & CB & G-CSF mobilized PB \\
\hline ABO matching & Match & Match & Match \\
Total nucleated cells & $1.93 \times 10^{\wedge} 7 / \mathrm{kg}$ & $2.22 \times 10^{\wedge} 7 / \mathrm{kg}$ & $13.6 \times 10^{\wedge} 8 / \mathrm{kg}$ \\
CD34-positive cells & $0.73 \times 10^{\wedge} 5 / \mathrm{kg}$ & $0.66 \times 10^{\wedge} 5 / \mathrm{kg}$ & $5.46 \times 10^{\wedge} 6 / \mathrm{kg}$ \\
Donor CMV-lgG status & negative & negative & positive \\
Conditioning & Flu/Mel/TBI & Flu/Bu & VP-16/ATG \\
GvHD prophylaxis & CsA+MTX & Tac+MTX & Tac+MMF+PTCy \\
Days from 1st transpntation & - & 38 & 84 \\
\hline
\end{tabular}

Abbreviations: HSCT, hematopoietic stem cell transplantation; CMV, cytomegalovirus; GvHD, graft-versus-host disease; MUD, matched unrelated donor; CB, cord blood; Flu, fludarabine; Mel, melphalan; TBI, total body irradiation; CsA, cyclosporine; MTX, methotrexate; MisUD, mismtched unrelated donor; Bu, busulfan; Tac, tacrolimus; MisRD, mismatched related donor; G-CSF, granulocyte-colony stimulating factor; PB, peripheral blood; VP-16, etoposide; ATG, anti-thymocyte globulin; MMF, mycophenolate mofetil; PTCy, posttransplant cyclophosphamide.

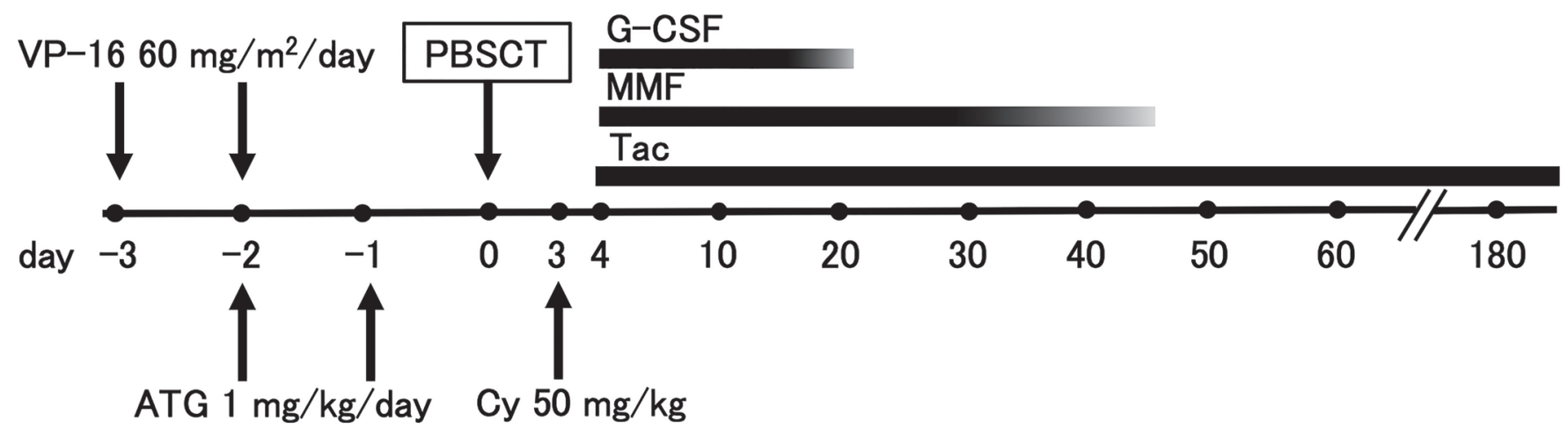

Figure 1. Treatment schema of haploidentical hematopoietic stem cell transplantation after graft failure

The patient underwent the third transplantation with peripheral blood stem cell from the haploidentical sibling donor after conditioning with 60 $\mathrm{mg} / \mathrm{m}^{2}$ of VP- 16 on days -3 and -2 , and $1 \mathrm{mg} / \mathrm{kg}$ of ATG on days -2 and -1 . In addition, for GvHD prophylaxis, $50 \mathrm{mg} / \mathrm{kg}$ of cyclophosphamide was administered on day +3 and Tac and MMF were initiated since day +4 .

VP-16, etoposide; PBSCT, peripheral blood stem cell transplantation; G-CSF, granulocyte-colony stimulating factor; MMF, mycophenolate mofetil; Tac, tacrolimus; ATG, anti-thymocyte globulin; Cy, cyclophosphamide.

cell count of $3.90 \times 10^{9} / \mathrm{L}$ with $1 \%$ myeloblasts, $0+$ promyelocytes, $5 \%$ myelocytes, $9 \%$ metamyelocytes, $49 \%$ neutrophils, and $27 \%$ lymphocytes along with tear drop epithelium and mild anemia. Biochemical tests revealed an increased lactate dehydrogenase (LDH) level of 483 U/L. Furthermore, bone marrow (BM) biopsy showed a hypoplastic BM with heteromorphic megakaryocytes and fibrosis without increased blast count. Cytogenetic analysis revealed a normal karyotype. Peripheral blood examination showed JAK2V617F mutation. Thus, his scores for both international prognostic scoring system and dynamic international prognostic scoring system for myelofibrosis were estimated to be intermediate-1.

After 5 months of ruxolitinib treatment, he complained of fatigue, with $\mathrm{LDH}$ level elevated to $1,451 \mathrm{U} / \mathrm{L}$ along with increased myeloblast count $(11 \%$ of white blood cell count), suggesting that his condition had progressed to leukemia. Because he had an only human leukocyte antigen (HLA) haploidentical sibling donor (brother) and no unrelated donor at a suitable timing, the first CBT was performed after conditioning with fludarabine, melphalan, and total body irradiation. HLA-antibodies were also negative. Unfortunately, the first transplantation resulted in graft failure; therefore, a second CBT was performed after conditioning with fludarabine and intravenous busulfan. Unfortunately, it also resulted in primary graft failure; therefore, a third transplantation was performed with peripheral blood stem cells from the haploidentical sibling donor and not the BM because of the necessity of more rapid hematopoietic recovery and the donor's intension. The conditioning regimen comprised etoposide $(60$ $\mathrm{mg} / \mathrm{m}^{2}$ on days -3 and -2 ) and rabbit anti-human thymocyte globulin $(1 \mathrm{mg} / \mathrm{kg}$ on days -2 and -1$)$, expecting suppression of cytokine production and T-cell immunosuppression (Table 1). In addition, for graft-versushost disease (GvHD) prophylaxis, cyclophosphamide $(50 \mathrm{mg} / \mathrm{kg})$ was administered on day +3 and tacrolimus and mycophenolate mofetil were initiated since day +4 (Figure 1).

The patient developed high fever associated with an 
allo-reactive response shortly after the transplantation, which improved immediately after the cyclophosphamide administration. Neutrophil engraftment (neutrophil count $>0.5 \times 10^{9} / \mathrm{L}$ ) was achieved on day +13 after the third transplantation without severe regimen-related toxicity and infection. Chimerism analysis was not performed. Cytomegalovirus (CMV) reactivation was detected using CMV antigenemia in neutrophils; thus, preemptive therapy with foscarnet was initiated, which resulted in the disappearance of CMV infection. Mycophenolate mofetil was discontinued at day +46 . On day +56 , skin stage 3 , gut stage 1 total grade II acute GvHD (aGvHD) developed, which required systemic administration of steroids (prednisolone $2 \mathrm{mg} / \mathrm{kg}$ ); this immediately relieved his symptoms, and prednisolone and tacrolimus doses were gradually tapered.

After discharge, platelet engraftment (platelet count $>$ $50 \times 10^{9} / \mathrm{L}$ ) was obtained on day +171 . On day +300 from the third transplantation, erythema appeared in $40 \%$ of the body surface area when oral prednisolone and tacrolimus were tapered to $0.2 \mathrm{mg} / \mathrm{kg}$ daily and a target trough level of 5-10 ng/mL, respectively. Therefore, he was diagnosed with cutaneous chronic GvHD (cGvHD) (NIH score 2). Increasing oral prednisolone and tacrolimus doses relieved his skin symptoms. Furthermore, systemic symptoms such as fatigue and splenomegaly improved, and he was alive and in fairly good health at 24 months after the third transplantation.

\section{Discussion}

The Center for International Blood and Marrow Transplant Research showed that the incidence of neutrophil engraftment after transplantation in PMF was 95\%, 73\%, and $83 \%$ among HLA-identical sibling, other related, and unrelated donors, respectively ${ }^{4}$. The Japan Society for Hematopoietic Cell Transplantation reported that the probability of neutrophil recovery on day +60 was significantly lower with unrelated BM transplantation (BMT) and CBT than with related BMT in patients with PMF $(P=0.015 \text { and } P=0.016 \text {, respectively })^{5}$. These large-scale retrospective studies indicated that graft failure is a complication frequently occurring in patients with PMF receiving allo-HSCT from an unrelated donor. Therefore, transplant physicians should recognize the possibility of graft failure and subsequently second HSCT in patients with myelofibrosis, unless HLAmatched sibling donor is available.

Recently, a retrospective study on clinical outcomes of haplo-HSCT with two-day PTCy to manage patients with graft failure showed that the median time to neutrophil engraftment was 18 days and the cumulative incidence of neutrophil engraftment at day 30 was $79 \%$. This study demonstrated the rescuable potential of salvage haplo-
HSCT with PTCy for graft failure, whereas HSCT complications accounted for $80 \%$ causes of deaths (with multiple organ failure as the leading cause). Owing to its high mortality, further studies with modified conditioning regimen and reduced PTCy dose may be necessary. In another retrospective study on clinical outcomes of haploidentical BMT using PTCy for hematologic malignancies in resistant relapse or in relapse after autologous transplantation, there was a trend toward a higher risk of extensive cGvHD among recipients of one-day PTCy (cyclophosphamide $50 \mathrm{mg} / \mathrm{kg}$ on day +3 ) than among those of two-day PTCy (cyclophosphamide $50 \mathrm{mg} / \mathrm{kg}$ on days +3 and +4$)^{7}$. Additionally, in a prospective study on the clinical outcomes of haploidentical peripheral blood stem cell transplantation (PBSCT) combined with unrelated cord blood using low-dose ATG with one-day PTCy, the cumulative incidences of grades II - IV and III - IV aGvHD were $19.4 \%$ and $6.9 \%$, respectively, whereas that of moderate-to-severe cGvHD within 6 months was $18.8 \%$, suggesting that a low-dose ATG with one-day PTCy was an effective regimen to prevent GvHD in haplo-PBSCT ${ }^{8}$. From the above studies, we used oneday PTCy and low-dose ATG, resulting in acceptable toxicities and GvHD. Therefore, one-day PTCy and lowdose ATG for GvHD prophylaxis might be an appropriate salvage treatment, particularly in patients who previously used multiple cytotoxic agents such as in the case of graft failure.

In conclusion, graft failure after allo-HSCT from unrelated donor is a relatively frequent complication in patients with myelofibrosis, and haplo-HSCT with oneday PTCy and low-dose ATG can be considered as one of the therapeutic options for graft failure. Furthermore, we hope that more haplo-HSCT cases with myelofibrosis will be accumulated as both primary allo-HSCT and salvage transplantation for graft failure.

\section{Acknowledgments}

We thank the nursing staff who cared for the patient at Kitakyushu Municipal Medical Center as well as Enago (www.enago.jp) for the English language review.

\section{Authors' Contribution}

H. I. and T. O. wrote the paper and S. O., Y. S., and Y. O. collected and managed the clinical data.

\section{Conflict of Interest}

The authors declare no conflict of interest. Disclosure forms provided by the authors are available here. 


\section{Financial Support}

No financial support.

\section{References}

1. Klampfl T, Gisslinger H, Harutyunyan AS, Nivarthi H, Rumi E, Milosevic JD, et al. Somatic mutations of calreticulin in myeloproliferative neoplasms. N Engl J Med. 2013; 369: 2379-90.

2. Laughlin MJ, Eapen M, Rubinstein P, Wagner JE, Zhang MJ, Champlin RE, et al. Outcomes after transplantation of cord blood or bone marrow from unrelated donors in adults with leukemia. N Engl J Med. 2004; 351: 2265-75.

3. Rocha V, Labopin M, Sanz G, Arcese W, Schwerdtfeger R, Bosi A, et al. Transplants of umbilical-cord blood or bone marrow from unrelated donors in adults with acute leukemia. N Engl J Med. 2004; 351: 2276-85.

4. Ballen KK, Shrestha S, Sobocinski KA, Zhang MJ, Bashey A, Bolwell BJ, et al. Outcome of transplantation for myelofibrosis. Biol Blood Marrow Transplant. 2010; 16: 358-67.

5. Murata M, Nishida T, Taniguchi S, Ohashi K, Ogawa H, Fukuda $\mathrm{T}$, et al. Allogeneic transplantation for primary myelofibrosis with BM, peripheral blood or umbilical cord blood: an analysis of the JSHCT. Bone Marrow Transplant. 2014; 49: 355-60.

6. Prata PH, Resche-Rigon M, Blaise D, Socié G, Rohrlich PS, Milpied N, et al. Outcomes of salvage haploidentical transplant with post-transplant cyclophosphamide for rescuing graft failure patients: a report on behalf of the francophone society of bone marrow transplantation and cellular therapy. Biol Blood Marrow Transplant. 2019; 25: 1798-1802.

7. Luznik L, O’Donnell PV, Symons HJ, Chen AR, Leffell MS, Zahurak M, et al. HLA-haploidentical bone marrow transplantation for hematologic malignancies using nonmyeloablative conditioning and high-dose, posttransplantation cyclophosphamide. Biol Blood Marrow Transplant. 2008; 14: 641-50.

8. Yang J, Jiang J, Cai Y, Li S, Wan L, Zhu J, et al. Low-dose antithymocyte globulin plus low-dose posttransplant cyclophosphamide as graft-versus-host disease prophylaxis in haploidentical peripheral blood stem cell transplantation combined with unrelated cord blood for patients with hematologic malignancies: a prospective, phase II study. Bone Marrow Transplant. 2019; 54: 1049-57.

https: //doi.org/10.31547/bct-2019-009

Copyright (C) 2020 APBMT. All Rights Reserved. 